\title{
Antioxidants used by Deinococcus radiodurans and implications for antioxidant drug discovery
}

\author{
Hong-Yu Zhang, Xue-Juan Li, Na Gao and Ling-Ling Chen
}

In his recent Opinion article (A new perspective on radiation resistance based on Deinococcus radiodurans. Nature Rev. Microbiol. 7, 237-245 (2009)) $)^{1}$, Michael Daly proposes that high levels of manganese can protect proteins from the reactive oxygen species (ROS) that are produced during irradiation. Owing to the close link between reactive oxygen species and various diseases, there is continuing interest in finding antioxidants as preventive or therapeutic drugs. According to the MDL Drug Data Report (MDDR) ${ }^{2}$, to date, seven antioxidant drugs have been launched, most of which (lipoic acid, policosanol, acetylcysteine, idebenone and probucol) are directly or indirectly derived from natural antioxidants. Exploring how organisms use antioxidants to combat ROS is therefore of great importance for antioxidant drug discovery.

It is well known that radiation-resistant bacteria have evolved strong antioxidant systems to survive ROS-mediated damage ${ }^{3}$. For example, various antioxidant enzymes, such as Mn- or Fe-superoxide dismutase, $\mathrm{Cu} / \mathrm{Zn}$-superoxide dismutase, catalase, peroxidase, thiol-alkyl hydroperoxide reductases, thioredoxin reductase, alkyl hydroperoxide reductase, peptide methionine sulphoxide reductase and glutaredoxin, have been identified from Deinococcus radiodurans, one of the most radiation-resistant bacteria ${ }^{3,4}$. Interestingly, Michael Daly revealed that nonenzymic Mn(II) complexes also have a crucial role in the antioxidant defence of $D$. radiodurans $^{1}$, which highlights the importance of small-molecule antioxidants in radiation resistance. This finding not only sheds new light on the radiation-resistant mechanisms of $D$. radiodurans but also has direct implications for antioxidant drug discovery. In fact, the development of $\mathrm{Mn}$ (II)-containing catalytic antioxidant drugs has attracted much attention in recent years ${ }^{5}$. However, as $D$. radiodurans contains a large number of metabolites, we speculate that other compounds might also be responsible for the antioxidant defence of $D$. radiodurans.

Through searching the Kyoto Encyclopedia of Genes and Genomes $(\mathrm{KEGG})^{6}$ and analysing the genome of D. radiodurans ${ }^{7}$, the biosynthetic pathways for some common antioxidant metabolites (for example, carotenoids, lipoic acid and folates) could be identified. It is interesting to note that the mutant of $D$. radiodurans that fails to synthesize carotenoids was more sensitive to ionizing radiation and hydrogen peroxide, and therefore that carotenoids, such as beta-carotene, lycopene and phytoene, indeed play an important part in the radiation resistance of $D$. radiodurans ${ }^{8}$. The antioxidant and radioprotective powers of lipoic acid have been widely recognized ${ }^{9}$, which strongly suggests that lipoic acid is also valuable in protecting $D$. radiodurans from oxidative damage. The radioprotective effect of folates was also observed ${ }^{10}$, which was linked, at least in part, to the antioxidant activity of folates ${ }^{10}$. Accumulating evidence indicates that folates are strong hydrogen and electron donors (when the proton is dissociated) ${ }^{11}$, especially in their reduced forms (for example, tetrahydrofolates), which endows them with substantial antioxidant potential that is similar to that of $\alpha$-tocopherol and ascorbic acid ${ }^{12}$. Moreover, the in vivo antioxidant effects of folates have been preliminarily recognized, which is independent of their well-known benefits to reduce homocysteine ${ }^{13}$. Thus, it is reasonable to infer that folates are involved in the antioxidant defence of $D$. radiodurans.
In summary, it seems that the antioxidant defence system of $D$. radiodurans consists of several lines of antioxidants, including enzymes and non-enzymatic small molecules, which is directly relevant to antioxidant drug discovery. Lipoic acid has been approved as an antioxidant drug, and therefore the other antioxidants used by $D$. radiodurans (that is, $\mathrm{Mn}$ (II) complexes and folates) are good starting points for finding novel antioxidant drugs.

Hong-Yu Zhang is at the Institute of Bioinformatics and National Key Laboratory of Crop Genetic Improvement,

College of Life Science and Technology, Huazhong

Agricultural University, Wuhan 430070, P. R. China.

Xue-Juan Li, Na Gao and Ling-Ling Chen are at the Shandong Provincial Research Center for Bioinformatic Engineering and Technique, Center for Advanced Study, Shandong University of Technology, Zibo 255049, P. R. China.

Correspondence to H.Y.Z. e-mail:zhy630@mail.hzau.edu.cn

1. Daly, M. J. A new perspective on radiation resistance based on Deinococcus radiodurans. Nature Rev. Microbiol. 7, 237-245 (2009).

2. MDL Drug Data Report (MDDR) (MDL Information Systems, San Leandro, 2004).

3. Wang, P. \& Schellhorn, H. E. Induction of resistance to hydrogen peroxide and radiation in Deinococcus radiodurans. Can. J. Microbiol. 41, 170-176 (1995). Makarova, K. S. et al. Genome of the extremely radiation-resistant bacterium Deinococcus radiodurans viewed from the perspective of comparative genomics. Microbiol. Mol. Biol. Rev. 65, 44-79 (2001).

5. Day, B. J. Catalytic antioxidants: a radical approach to new therapeutics. Drug Discov. Today 9, 557-566 (2004).

6. Kanehisa, M. \& Goto, S. KEGG: Kyoto Encyclopedia of Genes and Genomes. Nucleic Acids Res. 28, 27-30 (2000).

7. White, O. et al. Genome sequence of the radioresistant bacterium Deinococcus radiodurans R1. Science 286, 1571-1577 (1999).

8. Tian, B., Xu, Z., Sun, Z., Lin, J. \& Hua, Y. Evaluation of the antioxidant effects of carotenoids from Deinococcus radiodurans through targeted mutagenesis, chemiluminescence, and DNA damage analyses. Biochim. Biophys. Acta 1770, 902-911 (2007).

9. Manda, K., Ueno, M., Moritake, T. \& Anzai, K. a-Lipoic acid attenuates $\mathrm{X}$-irradiation-induced oxidative stress in mice. Cell Biol. Toxicol. 23, 129-137 (2007).

10. Pote, M. S., Gandhi, N. M. \& Mishra, K. P. Antiatherogenic and radioprotective role of folic acid in whole body gamma-irradiated mice. Mol. Cell Biochem. 292, 19-25 (2006).

11. Shen, L., Zhang, H.-Y. \& Ji, H.-F. A thermodynamic investigation of DPPH radical-scavenging mechanisms of folates. J. Mol. Struct. (Theochem) 856, 119-123 (2008).

12. Gliszczy $\square$ ska- $\square$ wigło, A. Folates as antioxidants. Food Chem. 101, 1480-1483 (2007).

13. Doshi, S. N. et al. Folate improves endothelial function in coronary artery disease: an effect mediated by reduction of intracellular superoxide? Arterioscler. Thromb. Vasc. Biol. 21, 1196-1202 (2001). 